\title{
Automation of Spacecraft Onboard Equipment Testing
}

\author{
Ludmila F. Nozhenkova, Olga S. Isaeva and Rodion V. Vogorovskiy \\ Institute of Computational Modelling SB RAS, Akademgorodok 50/44, 660036 Krasnoyarsk, Russia
}

\begin{abstract}
This article suggests an approach to automation of spacecraft onboard equipment testing, basing on a formalized sequence of scenarios determining measuring and control actions. We have developed a technology allowing to automate all stages of testing of spacecraft onboard systems' functional characteristics with the help of testing equipment's software. We have described the functions of software at the stages of test planning, preparation and execution, processing of the results and producing reports. This approach extends the possibilities to conduct experiments and allows to justify design solutions.
\end{abstract}

Keywords-spacecraft; onboard equipment; command and measuring system; testing; scenarios of testing; telemetry; telecommand packages

\section{INTRODUCTION}

Modern approaches to automation of onboard equipment testing are the base of spacecraft production development. Computer technique allows to increase the quality, reduce the prime cost and development time of onboard units.

Onboard equipment is a high-tech product with a long life cycle. The tests are aimed to verify the compliance of its functional parameters with the set requirements. This task is very difficult because the results of the tests on earth must guarantee the work of onboard equipment in space. [1]. In order to automate testing it is necessary to use special equipment and software that must be very safe, provide prompt data processing, comply with the modern requirements and have a flexible structure, adaptable for a specific use. Software must have a convenient interface, provide visualization of construction of complicated sequences of test procedures.

The study of the existing automation systems showed that each of them is aimed to solve a narrow range of tasks and, as a rule, has significant limits to extend many measurement functions. Different systems are realized in different software environments, thus making integration difficult, and sometimes impossible. There are products allowing integration of software modules, written in different languages, in order to complete the tasks of imitation, verification and testing. [2]. Besides, there are software and technological solutions allowing to set the methods of conducting functional control on the basis of formal specification of the tested systems. [3]. However, the tasks of adaption of the existing systems to the set study area, creation of interfaces of visual construction of test procedures, realization of the data reception and transfer control methods in accordance with the set standards in our case is impossible or extremely difficult. That's why the architecture, functionality and technologies of construction of the existing test systems were studied by the authors only in order to justify their own software solution.

As a result, we suggest our own approach to test automation. We have developed a technology and software allowing to automate all stages of spacecraft onboard equipment test preparation and execution.

\section{Test Automation TAsk SetTing}

The rules of different control objects' testing are determined by basic standards. For example, the ISO/IEC 9646 standard sets methods for communication systems' functional testing [4], The European Space Agency's standards determine the rules and order of spacecraft onboard equipment testing $[1,5]$, there also exist branch standards and methods for different companies. On the basis of the standards, test programs are developed and special software and hardware systems are created.

The authors had a task to create software for testing onboard control and measuring equipment of a spacecraft. The software must complete measurements and provide control over the command and measuring system's equipment by means of testing equipment.

The command and measuring system is one of the central systems of a spacecraft. It is designed to provide data exchange between earth and onboard control complexes. The earth control complex completes reception of telecommands, their primary processing and transfer to the onboard control complex, which, in its turn, sends packages of telemetry containing information about spacecraft onboard systems' function and the results of telecommands' processing. In order to unify the built solutions as data transfer protocols, the European Space Agency's standards are taken into consideration. The ESAPSS-04-107 standard [6] is designed for telecommand transfer, and the ESAPSS-04-106 standard [7] is designed for telemetry.

The command and measuring system is considered in our work as a black or grey box depending on the type of tests. The black box-type tests are conducted with the use of technical documentation, specification and other documents describing the requirements to the equipment's function. [8, 9]. Such tests are conducted in order to confirm electric high- and low-frequency characteristics of the command and measuring system. Most of the onboard equipment tests must be completed with control of telemetric information showing its condition. For testing reception and transfer of telecommands and telemetry, the tested object is presented as a grey box, where the structure and directions of data transfer are partially described. In this case, the command and measuring system is considered 
as a set of associated subsystems: transmitter, receiver and an interface module. The receiver accepts analog radio signal from the earth control complex, transforms it into a digital form and transfers it to the interface module for further processing. The transmitter completes the reverse procedure: accepts digital data package, transforms it into analog signal and transfers it to the earth control complex. The interface module completes data processing and interaction between onboard and earth control complexes of a spacecraft, including responding to different inquiries, as well as producing inquiries itself if necessary. The command and measuring system's graphical description is presented in Figure I.

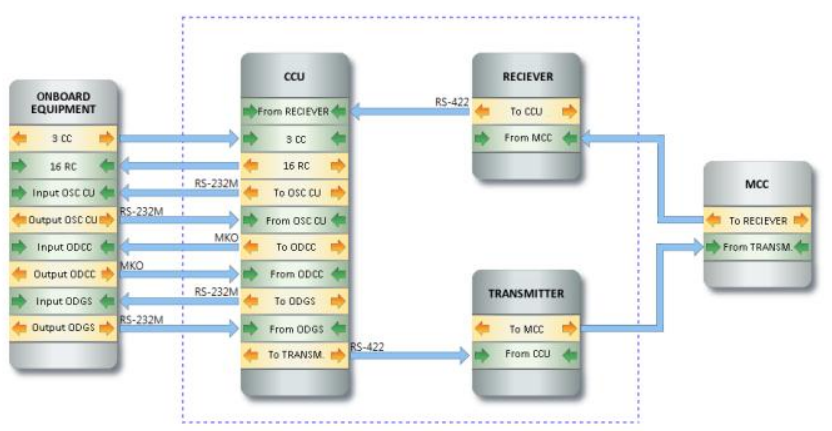

FIGURE I. THE TESTED OBJECT'S GRAPHICAL MODEL

The article Computer simulation of spacecraft onboard equipment [10] describes the software tools designed for building onboard equipment's models and its simulation modeling. Usage of simulation models allows to consider structure and methods of interactions between the tested object's subsystems in order to study their functioning features and to design tests. Meanwhile, in order to realize different types of tests of the command and measuring system we had to develop universal principles of description and conduction of the test procedures.

\section{TEST CONDUCTION AND ANALYSIS OF THE RESULTS}

We have designed a two-level architecture in order to create software for automation of onboard equipment test preparation and conduction. The lower level contains a library of software for the testing equipment. The software is completed in form of virtual devices (Virtual Instrument, VI), designed in the Labview environment [11]. The upper level is responsible for tuning the lower level's software libraries, creating measurement procedures, processing of the results of measurements.

In the basis of the upper level's software there lays the idea of a scenario. In this article the scenario is understood as a formal description of the series-parallel completion of measurements, methods of cycle setting, conditional transitions and the criteria of control and interruption of tests. The lower-level programs are described as "actions" in the scenario's formal description. The actions can be physical parameters' control functions, such as measurement of nominal frequency, determination of frequency and frequency instability and measurement of spectrum, as well as the functions of reception and transfer of data packages built in accordance with the ESA standards. The possibility to complete the sequence of actions together with the obligatory reception and control of telemetry showing the condition of the onboard systems is the important part of the suggested approach.

Development of the scenarios is completed with the help of a visual editor in the form of tables. The group of actions required to conduct a complex of measurements, are united into so-called "tasks" that can be used for building different testing scenarios. Application of such architecture allows to automate all testing procedures in order to analyze the work of a spacecraft's command and measuring onboard system's equipment. The designed software controls the work of the command and measuring equipment, completing each task and action stage-by-stage in accordance with the set testing scenario. In parallel, it provides control and analysis of the measured parameters of the tested object.

The software for testing onboard equipment is implemented at the company that develops sputnik systems. For implementation, we suggest a technological scheme of test execution (Figure II).

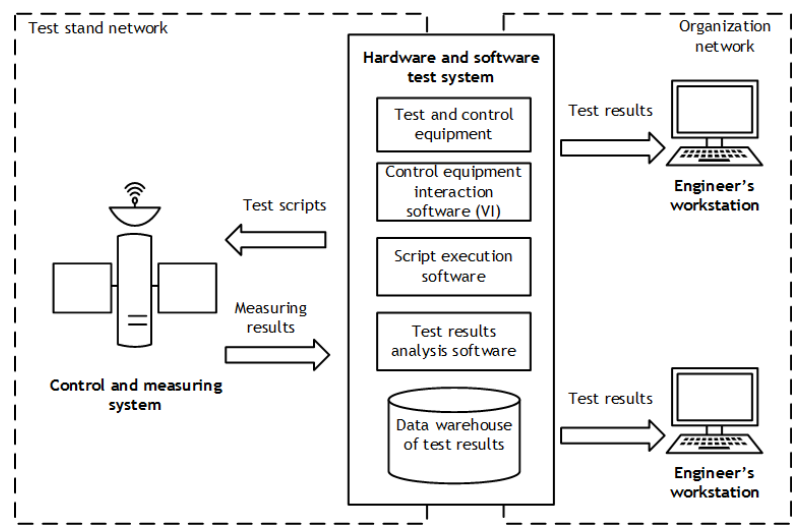

FIGURE II. TEST CONDUCTION ORGANIZATION SCHEME

Test conduction and analysis of the results are followed with visualization of the scenario's completion, succession and parameters of the testing procedures, checkpoints, the results of measurements. Also, the transferred and accepted data packages are shown. (Figure III).

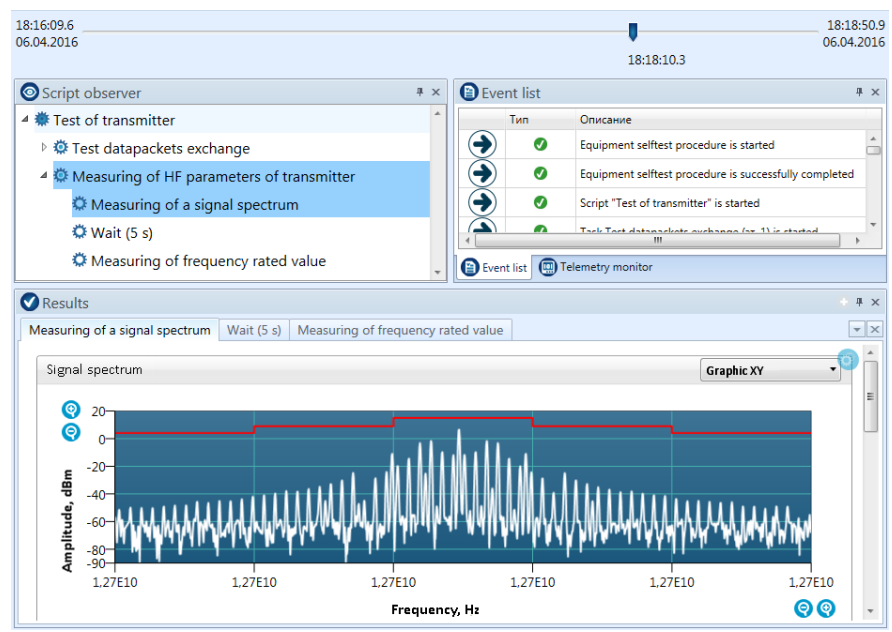

FIGURE III. VISUALIZATION OF THE TEST CONDUCTION 
The testing scenarios are completed automatically. The software provides constant control over the condition of the equipment and signals if anything goes wrong. In case of critical situations it makes a decision to stop testing. All events appearing during tests are written down and provided to the user. The results of the tests are brought into a report, providing details of the functional features of the tested object.

\section{SUMMARY}

The software created for automation of spacecraft onboard equipment testing allows to expand the possibilities to conduct experiments and makes it possible to make scientifically-based designer decisions. This software unites informational, measuring and computational functions. Interaction with the testing equipment allows to analyze the equipment's functional parameters and their accordance with the technical requirements.

The software is completed at the modern technical level and complies with the advanced trends of development of the testing systems. The software is oriented to work with the European telecommand and telemetry standards, which makes it topical, popular and competitive. It was tested by a company-developer of sputnik systems and received a positive feedback from onboard equipment designers.

\section{REFERENCES}

[1] ECSS-E-ST-10-02C. Space engineering - Verification - European Cooperation for Space Standardization (ECSS), 2009. 45 p.

[2] ECSS-E-ST-10-03C. Space engineering - Testing - European Cooperation for Space Standardization (ECSS), 2012. 128 p.

[3] Tretmans, J., Belinfante, A.: Automatic testing with formal methods. Enschede : University of Twente, Centre for Telematics and Information Technology (1999).

[4] Telemans, J.: Testing Concurrent Systems: A Formal Approach. CONCUR'99 Concurrency Theory. pp. 46-65. Springer-Verlag Berlin Heidelberg (1999).

[5] Garavel, H.: OPEN/CÆSAR: An open software architecture for verification, simulation, and testing. Tools and Algorithms for the Construction and Analysis of Systems. pp. 68-84. Springer Berlin Heidelberg (1998).

[6] ISO/IEC 9646-1: Information Technology - Open Systems Interconnection - Conformance testing methodology and framework - Part 1: General concepts, 1994. $46 \mathrm{p}$.

[7] ITU-T Recommendation Z.500 - Framework on formal methods in conformance testing, 1997. $49 \mathrm{p}$.

[8] Packet Telecommand Standard ESA PSS-04-107. Issue 2: European space agency (ESA), 1992. 166 p.

[9] Packet Telemetry Standard ESA PSS-04-106. Issue 1: European space agency (ESA), 1988. 73 p.

[10] Nozhenkova L., Isaeva O., Gruzenko E. Computer Simulation of Spacecraft Onboard Equipment // Proceedings series Advances in Computer Science Research (ISSN 2352-538x), Vol.18, P.943-945, DOI:10.2991/cisia-15.2015.

[11]LabVIEW function and VI reference manual. National Instruments Corporation, Austin, Texas, 1998. 